\title{
Crystallization of Proteins on Chip by Microdialysis for In Situ X-ray Diffraction Studies
}

\author{
Sofia Jaho $^{1}$, Niels Junius ${ }^{1,3}$, Franck Borel ${ }^{1}$, Yoann Sallaz-Damaz ${ }^{1}$, Jean-Baptiste Salmon ${ }^{2}$, Monika Budayova-Spano ${ }^{1}$ \\ ${ }^{1}$ Université Grenoble Alpes, CEA, CNRS, IBS ${ }^{2}$ CNRS, Solvay LOF, UMR 5258, Université Bordeaux ${ }^{3}$ ELVESYS SAS
}

\section{Corresponding Author}

Monika Budayova-Spano

monika.spano@ibs.fr

\section{Citation}

Jaho, S., Junius, N., Borel, F., SallazDamaz, Y., Salmon, J.B., BudayovaSpano, M. Crystallization of Proteins on Chip by Microdialysis for In Situ X-ray Diffraction Studies. J. Vis. Exp. (170), e61660, doi:10.3791/61660 (2021).

\section{Date Published}

April 11, 2021

\section{DOI}

$10.3791 / 61660$

URL

jove.com/video/61660

\section{Abstract}

This protocol describes the manufacturing of reproducible and inexpensive microfluidic devices covering the whole pipeline for crystallizing proteins on-chip with the dialysis method and allowing in situ single-crystal or serial crystallography experiments at room temperature. The protocol details the fabrication process of the microchips, the manipulation of the on-chip crystallization experiments and the treatment of the in situ collected X-ray diffraction data for the structural elucidation of the protein sample. The main feature of this microfabrication procedure lies on the integration of a commercially available, semipermeable regenerated cellulose dialysis membrane in between two layers of the chip. The molecular weight cut-off of the embedded membrane varies depending on the molecular weight of the macromolecule and the precipitants. The device exploits the advantages of microfluidic technology, such as the use of minute volumes of samples $(<1 \mu \mathrm{L})$ and fine tuning over transport phenomena. The chip coupled them with the dialysis method, providing precise and reversible control over the crystallization process and can be used for investigating phase diagrams of proteins at the microliter scale. The device is patterned using a photocurable thiolene-based resin with soft imprint lithography on an optically transparent polymeric substrate. Moreover, the background scattering of the materials composing the microchips and generating background noise was evaluated rendering the chip compatible for in situ X-ray diffraction experiments. Once protein crystals are grown on-chip up to an adequate size and population uniformity, the microchips can be directly mounted in front of the X-ray beam with the aid of a 3D printed holder. This approach addresses the challenges rising from the use of cryoprotectants and manual harvesting in conventional protein crystallography experiments through an easy and inexpensive manner. Complete $X$-ray diffraction data sets from multiple, isomorphous lysozyme crystals grown on-chip were collected at room temperature for structure determination. 


\section{Introduction}

Elucidating the three-dimensional (3D) structure of biological macromolecules is an unceasing pursuit in structural biology where X-ray crystallography remains the principal investigation technique. Applied for unraveling the structural details of complex macromolecules, such as proteins, it aims at facilitating the understanding of their mechanisms of actions and their involvement in various biological functions. Powerful X-ray sources at synchrotrons and X-ray freeelectron lasers (XFELs) provide all the tools required for a deeper insight into the proteins' structure at near atomic resolution. Despite the advantages that come along with the use of $\mathrm{X}$-rays for structural studies, there are intrinsic limitations to X-ray radiation and the crystallization process itself. Radiation damage provoked by high X-ray flux and long exposure times of the protein crystal in front of the $X$ ray beam are restrictive parameters that crystallographers have to surpass using cryogenic cooling ${ }^{1}$. However, finding the optimal cryocooling conditions can be laborious since conformational changes from the native protein structure or artifacts can be concealed ${ }^{2,3}$. Moreover, recent studies indicate that performing diffraction experiments at room temperature leads to lower specific radiation damage ${ }^{4}$. Another bottleneck in structural biology is the acquisition of well-diffracting crystals with a sufficient size ${ }^{5}$. Small crystals are easier to produce, especially in the case of membrane proteins, but are more susceptible to radiation damage even under cryocooling conditions because a high radiation dose must be directed in a smaller volume compared to the case of larger protein crystals ${ }^{6}$. The novel approach of serial crystallography ${ }^{7,8}$ at synchrotrons and XFELs can circumvent the restrains of radiation damage and at the same time exploit smaller crystals $(200 \mathrm{~nm} \text { to } 2 \mu \mathrm{m})^{7}$ by merging data sets from multiple, isomorphous and randomly oriented protein crystals and profiting from the associated technological advances such as femtosecond pulses, shorter exposure times and micro-focused X-ray beams ${ }^{5,7,9,10}$.

Microfluidic technology is valuable to X-ray crystallography, exhibiting manifold advantages for the crystallization of biological macromolecules and their structural investigation. Conducting crystallization experiments in microfluidic devices requires small volumes of protein sample, therefore constraining the production cost of these high-valued bio macromolecules and facilitating high-throughput screening and optimization of numerous crystallization conditions. Moreover, the inherent large surface area-to-volume ratio at the microfluidic scale and diffusion-limited transport phenomena enable fine control over flows and temperature or concentration gradients ${ }^{11,12,13,14}$, rendering microfluidic devices suitable for growing uniformly sized crystals and exploring phase diagrams ${ }^{15,16,17,18,19}$. Moreover, microfluidic tools display a distinctive potential to address another hurdle in protein crystallography, which is the sample delivery, and the necessity to handle and harvest protein crystals prior to their use for X-ray diffraction experiments. The method of on-chip and in situ X-ray crystallography eliminates the crystal manipulation and the potential deterioration of crystal quality prior to data collection. A wide range of microfluidic chips compatible for in situ Xray protein crystallography have been designed, developed, and tested by many research groups confronting the related restrictions arising from the nature of the microfabrication materials and their interactions with X-rays $\mathbf{s}^{14,19,20,21,22,23 .}$ The fabrication materials must be optically transparent, biologically inert and demonstrate high transparency to $\mathrm{X}$ - 
ray radiation and an optimal signal-to-noise ratio during data collection.

Most of the crystallization methods applied in conventional protein crystallography ${ }^{24,25}$ have also been implemented at the microfluidic scale 11,14 for on chip crystallization and in situ X-ray diffraction analysis. Simple, hybrid, or multi-layered microfluidic apparatus incorporating vapor diffusion $^{26}$, evaporation ${ }^{27}$, free interface diffusion (FID) ${ }^{28}$, microbatch $^{26}$, or even seeding ${ }^{29}$ have been used to crystallize soluble and membrane proteins. High throughput screening and optimization of crystallization conditions

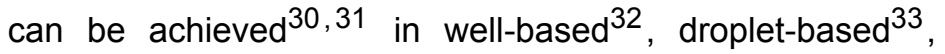
or valve-actuated ${ }^{34}$ devices. In situ X-ray diffraction experiments of challenging protein targets at room temperature have been conducted in microchips fabricated from various materials such as PDMS (polydimethylsiloxane), COC (cyclic olefin copolymer), PMMA (poly(methyl methacrylate) $)^{21,22,26,28,29}$, graphene films ${ }^{23}$, Kapton ${ }^{35}$, epoxy glue 6 , or NOA (Norland Optical Adhesive) ${ }^{19}$ and the materials' transparency to X-ray radiation and their contribution to background noise have been evaluated. Moreover, microchips have been designed to couple the in situ and the serial data collection strategies in a single tool for X-ray protein crystallography experiments at synchrotron sources $^{23,35,36}$ and XFELs ${ }^{7}$.

Room temperature in situ data collection has also been implemented in various delivery methods and devices. For example, Nogly et al. ${ }^{54}$ used a lipidic cubic phase (LCP) injector in order to study the structure of the light-driven photon pump bacteriorhodopsin (bR) by serial femtosecond crystallography (SFX) using an XFEL source. The crystal structure of bR was solved to $2.3 \AA$ resolution, demonstrating the compatibility of an LCP injector with time-resolved serial femtosecond crystallography (TR-SFX). Baxter et al. ${ }^{55}$ designed a high-density multi-crystal grid, fabricated by a 100 or $200 \mu \mathrm{m}$ thick polycarbonate plastic with laser-cut holes of various sizes. An additional $5 \mu \mathrm{m}$ thick polycarbonate film can be fixed to one side of the grid when using the device for sitting- or hanging-drop crystallization experiments. This highdensity grid can be used in multiple ways as crystals can be loaded directly onto the ports of the device or crystals can be grown on the device by vapor diffusion or the LCP method. Moreover, the grid can be adjusted in a standard magnetic base and used for in situ X-ray data collection at cryogenic or room temperature conditions. More recently, Feiler et al. ${ }^{56}$ developed a sample holder for macromolecular in situ Xray crystallography at cryogenic and ambient temperature with minimal background noise contribution. Specifically, the holder comprises of a plastic support, a transparent $\mathrm{COC}$ foil and a microporous structured polyimide foil. It was designed to replace the commonly used cover slides for setting up crystallization drops, while allowing in-place manipulation such as ligand soaking, complex formation, and cryogenic protection without opening the crystallization drop or manually handling the crystals. Moreover, the sample holder can be removed from the crystallization plate and placed onto a magnetic base for in situ data collection at standard goniometer-based beamlines. For ambient temperature data collection, the $\mathrm{COC}$ foil is removed prior to the experiment and only the $21 \mu \mathrm{m}$-thick polyimide foil contributes to background scattering, which in this case is minimal. These examples compose only a small fraction of the ongoing research and the multitude of versatile microchips developed for X-ray protein crystallography.

However, the dialysis protein crystallization method has not been widely incorporated within microfluidics. Dialysis is a diffusion-based method aiming for the equilibration 
of precipitant concentration through a semi-permeable membrane in order to approach the nominal concentration for protein crystallization and enables precise and reversible control over the crystallization conditions ${ }^{24}$. The Molecular Weight Cut-Off (MWCO) of the semi-permeable dialysis membrane can be chosen depending on the molecular weight of the macromolecule and the precipitants to allow the diffusion of small precipitant molecules while retaining the macromolecule of interest. Due to the reversibility of the dialysis process, it can be used in combination with temperature control to decouple and optimize nucleation and crystal growth independently ${ }^{37}$ for investigating phase diagrams by altering the precipitant concentration while using the same protein sample. The integration of membranes in microfluidics is reviewed by de Jong et al. ${ }^{38}$ and the case studies in biology implanting dialysis into microchips can be principally listed in sample preparation, concentration or filtration applications $39,40,41,42$ or cell-related studies 43,44 . Pervaporation through PDMS was used by Shim et al. ${ }^{37}$ to study the nucleation and growth of xylanase in various conditions. Water permeated through the $15 \mu \mathrm{m}$ thick PDMS membrane into the protein reservoir of the microfluidic device, subsequently altering the protein and precipitant concentration.

The protocol developed by Junius et al. ${ }^{19,45}$ for the fabrication of a microfluidic chip compatible for both onchip protein crystallization via microdialysis and in situ Xray diffraction experiments at room temperature is presented. The protocol for the device fabrication is directly inspired by the pioneering work accomplished by Studer and coworkers $^{12,46}$ for micro-patterned stickers of photo-curable thiolene-based resin NOA 81 embedding commercially available membranes, using soft imprint lithography. An innovative modification of the method resulted in microchips enabling the use of microdialysis to accurately monitor and control the experimental parameters for the on-chip growth of protein crystals and simultaneously exploit the advantages of microfluidics, such as reduced consumption of protein samples per experiment $(<1 \mu \mathrm{L})$. In a previous work, the principles of dialysis applied to a macro-scale system (typical volume $>20 \mu \mathrm{L}$ ) for screening and optimizing crystallization conditions by mapping temperature-precipitant concentration phase diagrams were demonstrated $^{47}$. In this work, a protocol is described for producing dialysis microchips incorporating regenerated cellulose (RC) dialysis membranes of different MWCO in order to perform crystallization assays on-chip and in situ X-ray diffraction data collection. The materials comprising the microchips have been evaluated for their transparency to X-rays ${ }^{19}$ and the devices can be set directly in front of the X-ray beam for room temperature in situ diffraction experiments, excluding the manual handling and minimizing the degradation of fragile protein crystals. In a case study, hen egg-white lysozyme crystals were grown onchip via microdialysis generating a uniformly sized population. The microchip was then mounted in front of the X-ray beam with a 3D-printed support ${ }^{19}$ and complete in situ diffraction data sets were collected at room temperature from multiple, isomorphous crystals, demonstrating the high potential and relevance of the chips for synchrotron serial crystallography studies of challenging macromolecular targets.

\section{Protocol}

\section{Mask design and master fabrication}

1. Draw the desired geometries of the microfluidic device using any vectorial drawing software. For each layer of the photoresist that will be used for the next step of photolithography, prepare an individual mask: one 
mask outlined with channels and pillars and one mask containing only the pillars.

2. Translate the CIF files generated by the drawing software into film photomasks. This can be done through commercial services. Require the appropriate photomask depending on the choice of photoresist used during photolithography.

NOTE: For the SU-8 photoresist, order masks with black features on a transparent background. SU-8 is an epoxybased negative photoresist, which means that during the exposure to UV light $(365 \mathrm{~nm})$ the parts exposed to UV are crosslinked whereas the rest remains soluble. Hence, all black patterns on the mask will not be cross-linked by UV light during the photolithography. Channels and pillars will be engraved on the masters.

3. Prepare two masters on silicon wafers for each chip's design by photolithography using SU-8 negative photoresist.

NOTE: Steps 1.3.1-1.3.7 are performed in a clean room. The steps described below are the traditional steps of photolithography followed by PDMS soft lithography, which are described in many textbooks. All the values of the experimental parameters (photoresist, time duration, temperature, etc.) depend on many subtle parameters and have to be optimized depending on the different apparatus used.

1. Use a 3-inch silicon wafer and treat the surface with plasma for $90 \mathrm{~s}$, in order to facilitate the deposition and attachment of the SU-8 photoresist.

2. Pour roughly $3 \mathrm{~mL}$ of SU-8 resist on the middle of the wafer and spin coat the SU-8 down to the desired thickness (Figure 1A). For $50 \mu \mathrm{m}$ nominal thickness, use SU-8 3050 and spin coat for $10 \mathrm{~s}$ at $500 \mathrm{rpm}$ and successively for $30 \mathrm{~s}$ at $3500 \mathrm{rpm}$. Soft bake the photoresist on a hot plate for $15 \mathrm{~min}$ at $368 \mathrm{~K}$ in order to be partially solidified by allowing the solvent contained in the resin to evaporate and prevent it from sticking on the photomask. Afterwards, leave the wafer at room temperature for $2 \mathrm{~min}$.

3. Expose the photoresist to UV light (Figure 1B). Use a mask aligner with a power of $35 \mathrm{~mW} \mathrm{~cm}^{-2}$ and 8 s exposure time.

4. Proceed with the post-exposure baking. Place the wafer on a hot plate for $5 \mathrm{~min}$ at $368 \mathrm{~K}$ to complete the photoreaction invoked by the UV exposure.

5. Remove all the SU-8 resist that was not crosslinked by placing the wafer in a bath containing propylene glycol methyl ether acetate (PGMEA) and stir for 15 min. Rinse the wafer with isopropanol until no blurry precipitation can be observed. Dry the wafer with nitrogen gas and store it in a Petri dish $(100 \mathrm{~mm} \mathrm{x}$ $15 \mathrm{~mm}$ standard size).

6. Treat the surface of the wafer with a silane in order to facilitate the detachment of polydimethylsiloxane (PDMS) that will be used to fabricate 2 stamps. Deposit the wafer on a tapped hot plate at 368 $\mathrm{K}$ for $10 \mathrm{~min}$ under the vapor atmosphere of hexamethyldisilazane (HMDS).

NOTE: If peeling off the PDMS from the wafer becomes difficult after several uses, the surface of the wafer should be treated again with HMDS vapor.

7. The first master containing the channels and the pillars is ready. Repeat these steps and prepare the second master patterning only the pillars.

NOTE: During photolithography, the aim is to obtain the geometries of the device on the SU-8 masters 
with a height of $50 \mu \mathrm{m}$. However, once the two SU-8 masters are fabricated, measure the height of the geometries engraved on the masters with a profilometer in order to acquire the experimental value. The measured value for both SU-8 masters fabricated for this protocol is approximately $45 \mu \mathrm{m}$.

\section{PDMS molds fabrication}

NOTE: The following steps of the protocol can be performed in any laboratory as long as a laminar flow hood is used, yellow light in the room is used when working with the NOA 81 resin (steps 3.6-3.11) and a source of UV light is available for polymerizing the NOA 81 resin (steps 3.7 and 3.11).

1. Prepare $50 \mathrm{~g}$ of PDMS silicone base and its curing agent in a 10:1 mass ratio.

2. Mix both the ingredients in a beaker with a spatula and place the mixture in a vacuum chamber to remove all the air bubbles.

3. Pour $25 \mathrm{~g}$ of the pre-mixed PDMS into the SU-8 master (stored in a Petri dish) patterning the channels and the pillars up to a height of approximately $5 \mathrm{~mm}$. Pour the remaining $25 \mathrm{~g}$ of the PDMS into the second SU-8 master patterning only the pillars up to a height of approximately $5 \mathrm{~mm}$ (Figure 1C).

4. Place both the Petri dishes in an oven and cure the PDMS layers at $338 \mathrm{~K}$ for $1 \mathrm{~h}$.

5. Cut the cured PDMS layer around the patterns of the SU-8 masters with a scalpel and gently peel off the PDMS molds from the masters (Figure 1D).

NOTE: The procedure described above, called replica molding, is frequently being used to prepare molds of PDMS that will be attached to glass surfaces and be part of a microfluidic device ${ }^{53}$. In this protocol, the
PDMS molds are not part of the chip, but they are used as intermediates for the chip fabrication. For each design, 2 PDMS molds are prepared from the respective SU-8 masters (Figures 1D and 1E) and will be used accordingly (as described below) for the fabrication of the microchip.

\section{Dialysis chip fabrication}

1. Place the PDMS mold patterning both the channels and pillars on a rigid microscope glass slide $(3 \times 1$ in. standard size) with the patterns facing upwards (Figure 1F). The central pillar that corresponds to the protein reservoir exceeds vertically by $45 \mu \mathrm{m}$ from the horizontal surface of the PDMS mold.

2. Cut and separate a dry piece of the regenerated cellulose (RC) dialysis membrane and deposit it on the central pillar of the PDMS mold, which is supported on the glass slide (Figure 1F).

NOTE: The RC dialysis membrane is commercially available, and the molecular weight cut-off (MWCO) is chosen accordingly with the molecular weight of the protein under study and the precipitants used. The size of the piece of the RC dialysis membrane depends on the design of the chip. In this protocol, 2 prototypes are designed where the volume of the protein reservoir is 0.1 or $0.3 \mu \mathrm{L}$. In these cases, the size of the dialysis membrane piece is $2 \times 2 \mathrm{~mm}^{2}$ or $4 \times 4 \mathrm{~mm}^{2}$, respectively.

3. Place the second PDMS mold patterning only the pillars facing downwards on top of the PDMS mold supported on the glass slide (Figure 1F). The central pillar of this mold corresponds to the protein reservoir and exceeds vertically (facing downwards) by $45 \mu \mathrm{m}$ from the horizontal surface. 
4. Align the central pillars of the 2 PDMS molds. The RC dialysis membrane is "sandwiched" in between the 2 PDMS molds (Figure 1G).

NOTE: The alignment between the microstructures of the 2 PDMS molds can be accomplished visually, without any extra equipment. Otherwise, this manipulation can be achieved under a microscope. A small shift between the reservoirs is not problematic, as long as the fluidic channel and the input or output points are not entirely covered.

5. Desiccate the assembly for $30 \mathrm{~min}$ in a vacuum chamber to remove all trapped air bubbles in the PDMS molds and to promote the insertion of the resin during the next step of the fabrication.

NOTE: The 2 PDMS molds are kept in place by PDMSPDMS adhesion and no extra pressure or other way of temporary bonding is required.

6. Fill the empty space between the 2 PDMS molds with the photocurable, thiolene-based resin NOA 81 by capillary imbibition (Figure 1H and 1I).

7. Cure the resin by exposure to UV light ( $365 \mathrm{~nm})$ for $8 \mathrm{~s}$ using a collimated UV lamp (power $35 \mathrm{~mW} \mathrm{~cm}^{-2}$ ).

NOTE: This first exposure allows NOA 81 resin to be partially crosslinked since a thin layer of NOA 81 in contact with the PDMS molds on both sides remains uncured.

8. Cut the excess of NOA 81 from the external sides of the PDMS molds with a scalpel.

9. Remove the upper PDMS mold with the partially crosslinked NOA 81 stuck on it from the bottom PDMS mold and the glass slide.

10. Cut a $175 \mu \mathrm{m}$ thick PMMA sheet in the standard dimensions of a microscope glass slide ( $3 \times 1$ inches) and peel off the plastic protection sheets from each side of the PMMA piece. Gently press the assembly of the upper PDMS mold and the partially cured NOA 81 on the PMMA piece (Figure 1J).

11. Cure again NOA 81 by exposure to UV light for $60 \mathrm{~s}$ and remove the upper PDMS mold (Figure 1K). The resin adheres to the PMMA substrate without any further treatment.

NOTE: The PDMS molds can be reused approximately up to 5 times after washing them with isopropanol and acetone, as long as the molds are not bent. The $\mathrm{RC}$ dialysis membrane is incorporated into the NOA 81 sticker and no further manipulation or mechanical clamping is required.

\section{Fluidic connectors}

NOTE: The design of the microfluidic chip consists of a linear fluidic channel for the crystallization solution and a central reservoir for the protein sample (protein reservoir), both shown from a top view of two microchips in Figure 2A. A RC dialysis membrane is embedded between these two microstructures (Figure 2D) and the crystallization process evolves while precipitants from the crystallization solution diffuse across the membrane due to a concentration gradient between the two compartments of the chip that are separated by the membrane. The microfluidic channel is imprinted on the bottom PDMS mold (Figure 1F). Once the fabrication protocol for the chips is completed, the linear channel is located on the bottom layer of the NOA 81 sticker in contact with the PMMA substrate, as shown in Figure 1K. An inlet and an outlet access point for the crystallization solution are located at each end of the linear channel and look like holes (total height $90 \mu \mathrm{m}$ ) as can be seen in Figure 2A. For the handling 
of the crystallization solution, connectors must be added on the access points.

1. Bond connectors that are commercially available (NanoPort) on the inlet and outlet of the microfluidic channel with fast epoxy glue (Figure 2C).

2. Choose the appropriate diameter of the PTFE tubes based on the size of the connectors. The PTFE tubes will be used for the introduction of the crystallization solution in the fluidic channel of the chip.

NOTE: Commercially available kits are recommended for easy and precise control over the flow rate and are usually combined with automated pressure-driven or flow controlled (syringe pumps) systems for mixing and fluid handling. However, the crystallization solution can be introduced manually into the linear channel with a disposable plastic syringe. In this case, steps 4.3 to 4.5 are suggested.

3. Fill a $1 \mathrm{~mL}$ disposable syringe with the crystallization solution. For the chips presented in this protocol, $400 \mu \mathrm{L}$ is sufficient in order to fill up the whole fluidic channel.

4. Cut two pipette tips so that the diameter of the tip at one side is equal to the inner diameter of the PTFE tube that will be used for the solution handling. Glue the cropped tips on the access points of the channel with fast epoxy glue (Figure 2B).

5. Connect the syringe with the cropped tips with a piece of PTFE tube of the appropriate size and introduce the solution within the channel by constantly pushing the syringe plunger slowly.

\section{Protein encapsulation}

NOTE: The pattern of the chip dedicated to being used as the protein reservoir remains so far open to the atmosphere. The following protocol is proposed to carefully confine the protein sample within the microfluidic chip.

1. Manually pipette a droplet of the protein sample inside the protein reservoir, located right upon the $\mathrm{RC}$ dialysis membrane, as illustrated in Figure 2E. The volume of the protein sample varies according to the design of the chip and can be 0.1 or $0.3 \mu \mathrm{L}$.

2. Apply a thin layer of high-vacuum silicone grease all around the protein reservoir.

3. Cut a small piece of a $175 \mu \mathrm{m}$ thick PMMA sheet and gently place it above the thin layer of the silicone grease. The PMMA piece must cover the whole surface of the protein reservoir where the protein solution is deposited. NOTE: The silicone grease is used to enhance airtightness and prevent the spreading of the protein droplet. There is no bonding or sealing between the PMMA piece used for covering the protein reservoir and the NOA 81 sticker. The contact between them is a solid/ solid interface. In order to produce an overall sealing and an air-tight encapsulation of the protein sample, a piece of Kapton tape is used as described in step 5.4.

NOTE: It is sometimes difficult to confine the protein sample within the dedicated cavity of the device (protein reservoir) when a pressure-driven system is used for the introduction of the crystallization solution within the fluidic channel (step 6.4). To avoid the problem mentioned above, relatively low pressure values should be maintained while circulating the crystallization solution. An injection pressure of 20-60 mbar for aqueous solutions or 50-150 mbar for more viscous solutions (PEGs, glycerol) is suggested ${ }^{19}$.

4. Cut a piece of Kapton tape (20 $\mu \mathrm{m}$ thick) large enough to cover the PMMA piece set above the protein reservoir 
and to stick on the NOA chip around all edges. The protein sample is encapsulated within the reservoir and the chip is ready to be used for the crystallization experiment, as shown in Figure 2C.

NOTE: The chips can be reused several times as long as the dialysis membrane and the adhesion of NOA on the PMMA substrate are not deteriorated. If these parts of the chip are damaged, leaks are observed verifying that the device can no longer be used. Washing the chips depends on the crystallization solution. In the case of low viscosity solutions (salts, buffers), the fluidic channel can be washed by merely introducing distilled water and let it flow for a few minutes. $400 \mu \mathrm{L}$ is the volume required in order to completely exchange a solution within the channel with another solution. In the case of more viscous solutions (PEGs, glycerol), reusing the chips is not recommended since washing the channel only with water is not sufficient. The upper part of the chip, where the protein reservoir is located, can also be washed with distilled water and dried with pressurized air.

\section{On-chip protein crystallization}

1. Weigh lyophilized hen egg-white lysozyme powder and dissolve in distilled water to obtain a final concentration of $30 \mathrm{mg} \mathrm{mL}^{-1}$.

2. Filter the protein solution through a $0.22 \mu \mathrm{m}$ filter and centrifuge for $5 \mathrm{~min}$ at the highest speed at $293 \mathrm{~K}$ to remove all solid particles. Use the supernatant for the crystallization experiment.

3. Prepare $500 \mu \mathrm{L}$ of crystallization solution containing the buffer and the precipitant in concentrations provided in Table 1. Filter the solution through a $0.22 \mu \mathrm{m}$ filter.
4. Inject the solution into the inlet point of the chip with a syringe or an automated pressure-driven fluidic system or a syringe pump, as described in steps 4.1-4.5.

NOTE: The crystallization experiment can occur either under static condition, if the microfluidic channel is filled with the crystallization solution and set aside, or under flowing conditions, if it is continuously injected inside the channel at a constant flow rate. For the latter case, the use of an external pressure-driven system or syringe pump is recommended. The realization of the experiment under flowing conditions also provides the possibility to dynamically exchange crystallization solutions within the fluidic channel. Thus, multiple experiments can be conducted while using the same protein sample.

5. Once the fluidic channel is filled with the crystallization solution, seal the inlet and outlet ports of the chip with parafilm tape.

6. Pipette the appropriate volume of the protein solution within the protein reservoir and encapsulate the protein sample as described in steps 5.1-5.4.

7. Store the chip at $293 \mathrm{~K}$.

NOTE: Crystallization via dialysis follows a different kinetic trajectory from experiments conducted with the vapor diffusion method or batch crystallization and depends profoundly on the nature of precipitant molecules involved in the diffusion process, according to measured data ${ }^{51}$, and it might take more time for the nucleation to start. In order to prevent evaporation, if any, during this period, place the chip in a humidity saturated atmosphere at $293 \mathrm{~K}$.

\section{In situ and on-chip X-ray diffraction}

1. 3D printed support for beamlines 
1. Print the support that can carry up to three chips simultaneously. The dimensions of the support are the same as the dimensions of commercial crystallization plates (96 well/SBS standard) compatible with in plate $\mathrm{X}$-ray diffraction experiments.

NOTE: The printing of the support can be assigned to commercial services. The support was designed using a $3 D-C A D$ designing software and is shown in Figure 3A during on-chip protein crystallization experiments and in Figure 3B during in situ X-ray diffraction data collection at BM30A-FIP (ESRF).

2. Stabilize the dialysis chips on the support with a single- or double-sided tape.

2. In situ X-ray diffraction

1. Collect X-ray diffraction data at room temperature from crystals grown in the protein reservoir. For instance, use X-rays with an energy of $12.656 \mathrm{keV}$, a flux of $3.32 \times 10^{10}$ photons $\mathrm{s}^{-1}$ and a beam size of $250 \times 250 \mu \mathrm{m}^{2}$. Record the diffraction images with an ADSC Quantum 315r detector with a matrix of 3 x 3 CCD for an active surface of $315 \times 315 \mathrm{~mm}^{2}$ and 9.4 mega pixels resolution.

NOTE: The diffraction data for the lysozyme crystals grown on the dialysis chips were collected at BM30A-FIP beamline at the European Synchrotron Radiation Facility (ESRF). However, the beam size, the flux and the detector type may be different in other X-ray radiation sources. The 3D printed support enables data collection with an angular range of $-40^{\circ}$ to $+40^{\circ}$ around the crystal. The number of the lysozyme crystals exposed for in situ data collection, the number of the diffraction patterns collected for each crystal, the oscillation angle range per exposure and the exposure time are summarized in Table 2.

3. Data treatment

1. Process the complete or partial data sets with the diffraction patterns for the lysozyme crystals with the XDS program ${ }^{48}$.

2. Generate the HKL file for each data set and scale them using the XSCALE software ${ }^{48}$.

3. Use molecular replacement with the program Phaser of the CPP4 suite ${ }^{49}$ and determine the phases for model building. For this step, use the known 3D coordinates of lysozyme from the Protein Data Bank (PDB) entry 193L.

4. Refine the structure using Phenix ${ }^{52}$ and inspect the final protein model using $\mathrm{COOT}^{50}$. 


\section{Representative Results}

The microfluidic chips developed by Junius et al. ${ }^{19,45}$ are compatible for both on-chip protein crystallization with the microdialysis method and in situ X-ray diffraction data collection at room temperature. Pictures of the microchips, their detailed design, the fluidic connectors, and the $\mathrm{RC}$ dialysis membrane are illustrated in Figure 2. The crystallization experiments are set up by manually pipetting the protein sample directly into the protein reservoir and introducing the crystallization solution into the linear fluidic channel with an automated pressure-driven system or syringe pump or manually with the aid of a syringe. The protein reservoir and the fluidic channel can be distinguished in Figure 2A. Designs for fabricating chips with $0.1 \mu \mathrm{L}$ or 0.3 $\mu \mathrm{L}$ maximum volume of the protein reservoir are shown in Figure $2 \mathrm{~A}$ on the left and right, respectively. Chips with a $0.2 \mu \mathrm{L}$ or $0.7 \mu \mathrm{L}$ maximum capacity for the protein sample are shown elsewhere ${ }^{19}$. The highlight of the protocol for the device fabrication can be narrowed down on the use of the photocurable thiolene-based resin NOA 81 embedding commercially available RC dialysis membranes of various MWCOs. During the fabrication of the microfluidic devices, the linear fluidic channel is imprinted on the bottom PDMS mold (Figure 1F), while the upper PDMS mold consists only of the patterned pillars for the protein reservoir and the inlet and outlet ports (Figure 1F). Once NOA 81 is crosslinked and the PDMS molds are removed from the assembly (Figure $1 \mathrm{~K})$, the fluidic channel is located at the bottom layer of the microchip and the protein channel and inlet/outlet ports are located on both layers. Figure $1 \mathrm{~L}$ illustrates a side view schematic of the dialysis chip where all the layers of the device and their respective thickness are indicated. The height of the patterns imprinted on the bottom layer of the chips (fluidic channel) is approximately $45 \mu \mathrm{m}$, while the total height of the inlet and outlet ports is approximately $90 \mu \mathrm{m}$. The protein reservoir (45 $\mu \mathrm{m}$ height) is also illustrated in Figure 2D and 2E. The alignment of the two layers was investigated under an optical microscope and the piece of the RC dialysis membrane incorporated within the microchip can be clearly distinguished in Figure 2D. In the same figure, air has been entrapped within the fluidic channel during the injection of the crystallization solution, as can be seen in the upper-left part of the protein reservoir. Figure $\mathbf{2 E}$ is a close-up photograph of the protein reservoir after the manual deposition of the protein droplet with a pipette and before the encapsulation of the droplet with a piece of PMMA and Kapton tape, as described in steps 5.2 and 5.3 of the protocol. The microfluidic chip ready to be used for crystallization experiments, after the encapsulation of the protein sample and the gluing of the fluidic connectors, is depicted in Figure 2C. The air-tight assembly ensures that leakages cannot occur. The fluidic connectors for the inlet and outlet ports of the microfluidic channel can be either the commercially available ones as described in step 4.1 of the protocol and shown in Figure 2C, or disposable laboratory pipette tips can be used for the same purpose (Figure 2B, protocol step 4.4). 
For the fabrication of the microfluidic chips, optically transparent and biologically inert materials were chosen, demonstrating high compatibility for in situ X-ray diffraction experiments at room temperature. The interactions of $X$ rays, absorption and scattering, with the materials composing the microfluidic device and the surrounding atmosphere (air) generate a signal known as background noise. This noise sums up to the diffraction signal of the protein crystals recorded by the detector, decaying the signal-to-noise ratio and should be maintained as low as possible during X-ray diffraction data collection. We have evaluated the background noise generated by the materials comprising the protein reservoir, which is in the direct path of the X-ray beam. The protein reservoir consists of the RC dialysis membrane, the Kapton tape and two PMMA pieces, one used as a substrate for the microchip and one used for the encapsulation of the protein sample. The thickness of the PMMA is $2 \mathrm{x}$ $175 \mu \mathrm{m}$, of the Kapton tape $20 \mu \mathrm{m}$, and the RC dialysis membrane is approximately $40 \mu \mathrm{m}$ thick (Figure 1L). The total thickness of these layers is about $410 \mu \mathrm{m}$ and the NOA 81 layer is not in the direct X-ray path. Apart from the thickness of the fabrication materials, their density is also crucial for measuring the background scattering noise, as X-ray scattering increases with the elemental atomic number. For this reason, helium flux (a feature provided at BM30A-FIP at ESRF) was used instead of air during the data collection for material characterization and for protein diffraction experiments. Figure $\mathbf{3 C}$ illustrates the background noise generated by the Kapton tape, the RC dialysis membrane, the PMMA sheet, and their assembly in helium atmosphere. Each material was exposed for 20 s to X-rays of $0.98 \AA$ wavelength and the sample-detector distance was $200 \mathrm{~mm}$. The experiments were performed at the BM30AFIP beamline at ESRF, as explained in step 7 of the protocol. Diffuse rings attributed to the interactions of the X-ray beam with the materials can be distinguished for the Kapton tape at a resolution lower than $4 \AA$, the PMMA sheet between 4-8 $\AA$, and the dialysis membrane between 4-5 $\AA$ resolution. The background noise generated by the dialysis chip is mainly observed at a resolution lower than $6 \AA$ that does not affect the treatment of high-resolution diffraction data of the large lysozyme crystals. The background scattering intensity as a function of the resolution for the microchip and the separate materials are shown elsewhere ${ }^{19}$. In the measurement presented in Figure 3C, the dialysis chip was empty of any solution (protein or precipitant solution) and the contribution of the presence of solution to the background noise has not been measured. The chips were mounted in front of the X-ray beam with a 3D-printed support (Figure 3B) designed for in situ diffraction experiments ${ }^{19}$. However, the same support with dimensions equal to the dimensions of a 96-well/SBS standard crystallization plate, can be used for performing 1 to 3 crystallization experiments concurrently, as it can hold up to 3 chips simultaneously (Figure $3 \mathrm{~A}$ ). 
Experiments were conducted to evaluate the efficiency of the microfluidic devices for the on-chip crystallization of model soluble proteins with the microdialysis method. The fluidic channel was filled as described in step 4 of the protocol, while steps 5 and 6 described how to encapsulate the protein sample within the dedicated protein reservoir and how to set up the crystallization experiments. Figure 4 shows lysozyme crystals grown at $293 \mathrm{~K}$ under $1.5 \mathrm{M}$ sodium chloride $(\mathrm{NaCl})$ with $0.1 \mathrm{M}$ sodium acetate $\left(\mathrm{CH}_{3} \mathrm{COONa}\right) \mathrm{pH} 4.0$ (A) and under $1 \mathrm{M} \mathrm{NaCl}, 0.1 \mathrm{M} \mathrm{CH}_{3} \mathrm{COONa} \mathrm{pH} 4.5$ with $30 \%$ polyethylene glycol (PEG) 400 (B). The lyophilized lysozyme powder was dissolved in water to a final concentration of $\sim 30 \mathrm{mg} \mathrm{mL}^{-1}$ or in $20 \mathrm{mM} \mathrm{CH} 3 \mathrm{COONa} \mathrm{pH} 4.2$ buffer to a final concentration of $\sim 20 \mathrm{mg} \mathrm{mL}^{-1}$ for the experiments illustrated in Figures 4A and 4B, respectively. The volume of the protein sample in both experiments was about 0.3 $\mu \mathrm{L}$ and the MWCO of the RC dialysis membrane embedded within the microchips lies in the range of $6-8 \mathrm{kDa}$. The lysozyme crystals shown in Figure $\mathbf{3 A}$ grew within $1 \mathrm{~h}$ and the crystals in Figure 3B grew within $30 \mathrm{~min}$ from the onset of the experiment. The crystallization experiments were carried out under static conditions. However, it has been shown ${ }^{19}$ that conducting the experiments under flowing conditions provides the possibility to dynamically exchange the crystallization conditions and study phase diagrams, verifying the reversibility of the microdialysis method.
In situ X-ray diffraction data from the lysozyme crystals shown in Figure 4A were collected to demonstrate the suitability of the dialysis chips for such experiments. The data collection was carried out at BM30A-FIP beamline (ESRF) at room temperature, as described in step 7.2.1 of the protocol. The microchips were mounted at the beamline with the aid of the 3D-printed support (Figure 3B) and complete X-ray diffraction data sets were collected from two single lysozyme crystals grown on-chip under the conditions given in the second line of Table 1. The observed reflections of the data sets were processed, indexed, and integrated using $\mathrm{XDS}^{48}$ and the molecular replacement and refinement were accomplished using Phaser ${ }^{49}$ and Phenix ${ }^{52}$, respectively. The crystallographic statistics for the complete data set of each lysozyme crystal and for the merging of the two data sets are provided in Table 2. For the molecular replacement, the PDB entry $193 \mathrm{~L}$ was used.

Electron density maps from a single lysozyme crystal and the merged data set of the two crystals have been obtained at $1.95 \AA$ and $1.85 \AA$, respectively, and are illustrated in Figures 5A and 5B. Both electron density maps show detailed structural information that can be obtained by in situ X-ray diffraction experiments conducted directly on the dialysis microchip at room temperature from a single crystal or from multiple crystals, rendering the chips compatible for in situ X-ray crystallography studies. 
A

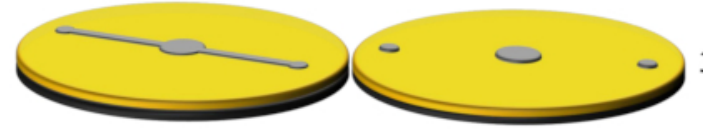

C

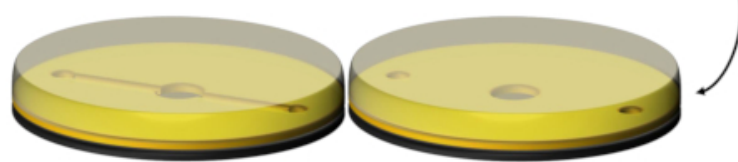

Apply PDMS on
SU-8 master

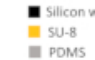

D

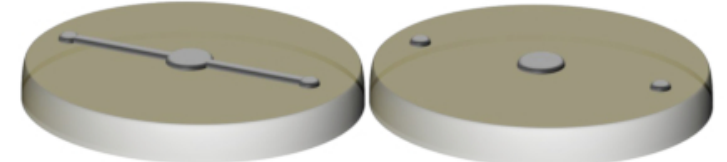

E
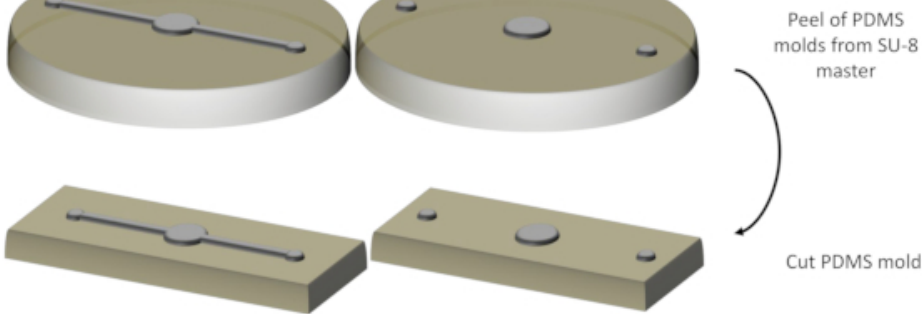

Cut PDMS molds
F

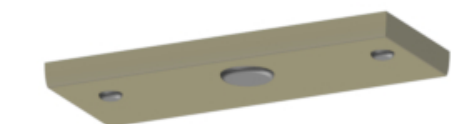

G
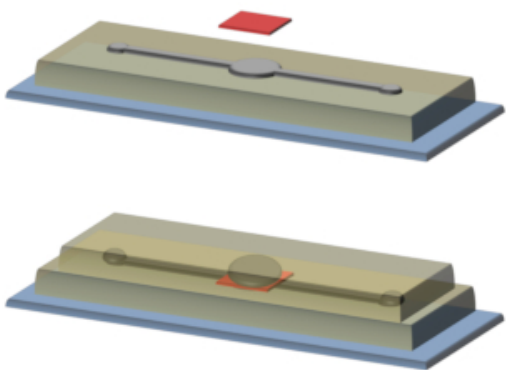

H
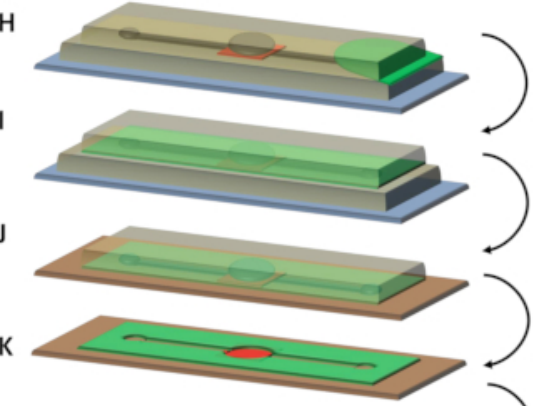

L

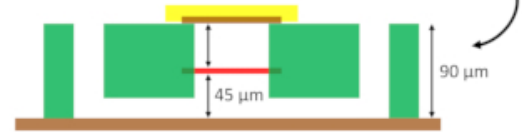

Deposit PDMS molds on glass slide. Cut and place $\mathrm{RC}$ dialysis membrane in between the central pillars.

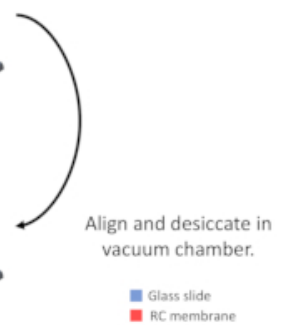

Deposit NOA81 resin.

Fill in with NOA81 by capillarity. $1^{\text {st }}$ UV exposure.

Remove lower PDMS mold. Deposit on PMMA.

$2^{\text {nd }}$ UV exposure.

Remove upper PDMS mold.

Side view of the dialysis chip.

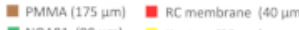
- NOAB1 $(90 \mu \mathrm{m})=$ Kapton $(20 \mu \mathrm{m})$

Figure 1: Schematic illustration of the dialysis chip fabrication. (A) SU-8 resin is deposited on two silicon wafers and spin coated. (B) A SU-8 master is acquired after irradiating the photoresist with UV light through a photomask and 
developing the unexposed parts. (C) PDMS is dispensed upon the SU-8 masters and after being cured at $338 \mathrm{~K}$ for $1 \mathrm{~h}$, (D) the 2 PDMS molds produced by replica molding and imprinting the micro-patterns are peeled of the masters and (E) cut to the appropriate size. (F) The PDMS molds are supported on a glass slide incorporating the RC dialysis membrane in between the two central pillars. (G) The 2 PDMS molds are then aligned and desiccated for $\sim 30$ min in a vacuum chamber. (H) The NOA 81 resin is poured in between the two molds and (I) fills the space by capillarity. (J) After the first exposure to UV light, the bottom PDMS mold is removed and the assembly is deposited on a PMMA sheet. (K) The second UV exposure follows in order to fully polymerize the NOA 81 resin and the dialysis chip is ready-to-use after removing the remaining upper PDMS mold. (L) Side view schematic of the dialysis chip where all the layers of the device and their respective thickness are indicated. Please click here to view a larger version of this figure.
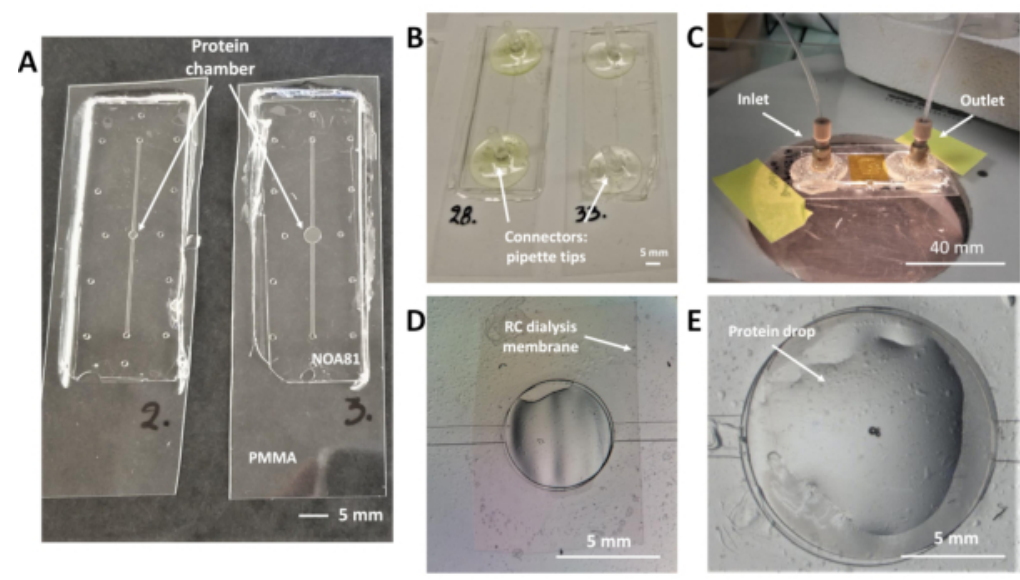

Figure 2: Dialysis chips embedding an RC dialysis membrane for on-chip protein crystallization and in situ X-ray diffraction experiments. (A) NOA 81 microchips on $175 \mu \mathrm{m}$ thick PMMA substrate with a protein reservoir of $0.1 \mu \mathrm{L}$ (left) and $0.3 \mu \mathrm{L}$ (right) nominal volume. (B) Microchips with pipette tips as fluidic connectors glued on the inlet and outlet ports of the fluidic channel. (C) Picture of a microchip during a crystallization experiment. The protein sample is encapsulated with a piece of $175 \mu \mathrm{m}$ thick PMMA sheet and Kapton tape. Peek Nanoport connectors are used for the inlet and outlet ports of the fluidic channel. (D) Top view of the protein reservoir during the circulation of the crystallization solution within the fluidic channel. Air is trapped in the upper part of the reservoir right under the RC dialysis membrane, which can be clearly detected. (E) Top view of the dialysis reservoir through an optical microscope during the deposition of the protein sample. The protein droplet is deposited right above the embedded RC dialysis membrane. Please click here to view a larger version of this figure. 

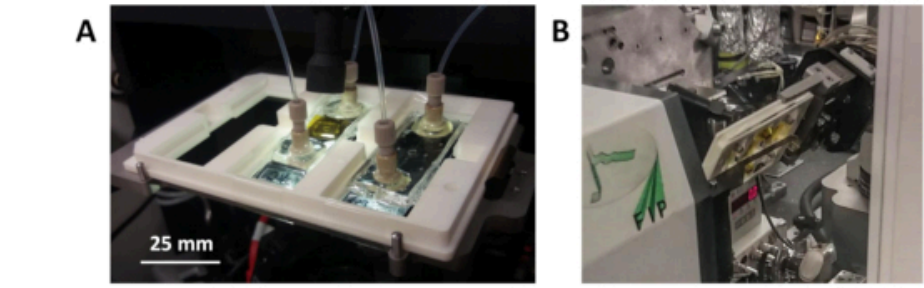

C

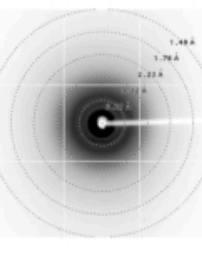

Kapton

RC dialysis membrane

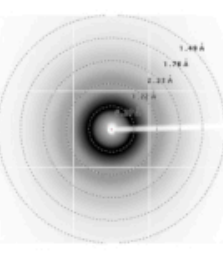

PMMA

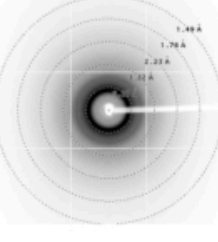

Dialysis chip

Figure 3: The 3D-printed support (A) for the microchips used during crystallization experiments and (B) mounted in front of the X-ray beam at BM30A-FIP beamline at the ESRF for in situ X-ray diffraction experiments. (C) Background noise generated by the interaction of X-rays with Kapton, RC dialysis membrane, PMMA, and the dialysis chip (from left to right). Please click here to view a larger version of this figure.
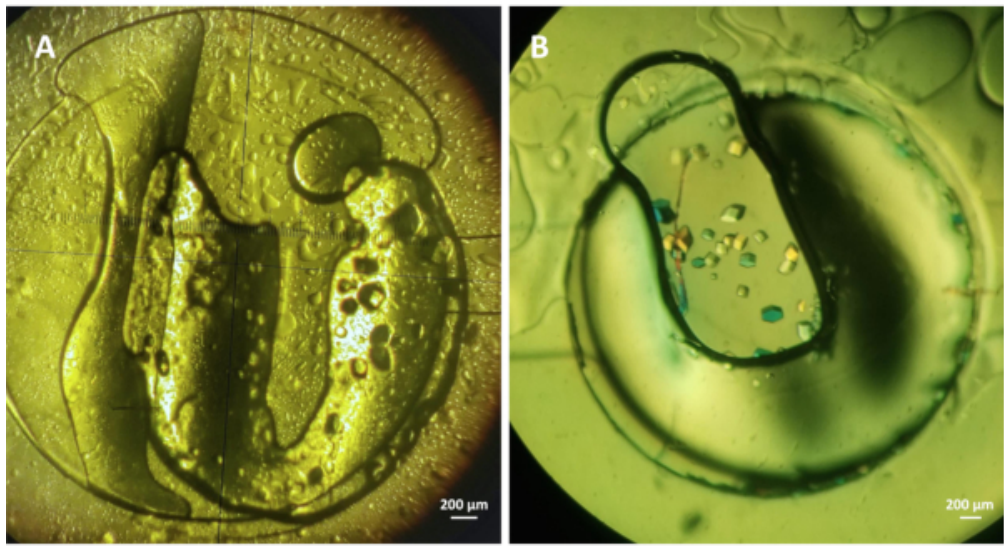

Figure 4: On-chip crystallization of lysozyme with the microdialysis method. (A) Lysozyme $\left(\sim 30 \mathrm{mg} \mathrm{mL}^{-1}\right) \mathrm{crystals}^{\mathrm{s}}$ grown on-chip under $1.5 \mathrm{M} \mathrm{NaCl}$ and $0.1 \mathrm{M} \mathrm{CH}_{3} \mathrm{COONa} \mathrm{pH} 4.0$ and $(\mathbf{B})$ lysozyme $\left(\sim 20 \mathrm{mg} \mathrm{mL}^{-1}\right)$ crystals grown under crystallization conditions containing $1 \mathrm{M} \mathrm{NaCl}, 0.1 \mathrm{M} \mathrm{CH} 3 \mathrm{COONa} \mathrm{pH} 4.5$, and 30\% PEG 400. Both experiments were conducted at $293 \mathrm{~K}$. Please click here to view a larger version of this figure. 
A

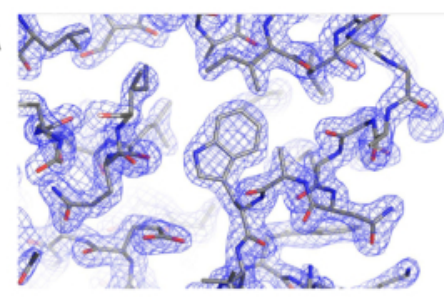

B

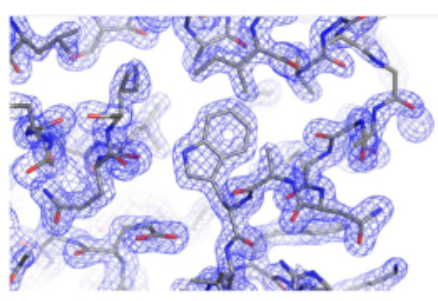

Figure 5: Electron density maps of the refined lysozyme structure from (A) a single crystal and (B) the merged data set of two crystals grown on-chip via microdialysis. The maps were obtained at $1.95 \AA$ and $1.84 \AA$, respectively, contoured at $1 \sigma$. Please click here to view a larger version of this figure.

\begin{tabular}{|c|c|c|c|c|c|}
\hline Protein & $\begin{array}{l}\text { Protein } \\
\text { concentration } \\
\left(\mathrm{mg} \mathrm{mL}^{-1}\right)\end{array}$ & Protein bufffer & $\begin{array}{l}\text { Initial concentration of } \\
\text { precipitant solution }\end{array}$ & $\begin{array}{l}\text { MWCO of RC } \\
\text { dialysis } \\
\text { membrane } \\
\text { (kDa) }\end{array}$ & $\begin{array}{c}\text { Temperature } \\
\text { (K) }\end{array}$ \\
\hline Lysozyme & $\sim 30$ & Water & $\begin{array}{c}1.5 \mathrm{M} \mathrm{NaCl} \\
0.1 \mathrm{M} \mathrm{CH}_{3} \mathrm{COONa} \mathrm{pH} 4.0\end{array}$ & $6-8$ & 293 \\
\hline Lysozyme & $\sim 20$ & $20 \mathrm{mM} \mathrm{CH}_{3} \mathrm{COONa} \mathrm{pH} 4.2$ & $\begin{array}{c}1 \mathrm{M} \mathrm{NaCl} \\
0.1 \mathrm{M} \mathrm{CH}_{3} \mathrm{COONa} \mathrm{pH} 4.5 \\
30 \% \text { PEG } 400\end{array}$ & $6-8$ & 293 \\
\hline
\end{tabular}

Table 1: Composition of the protein buffer and the precipitant solution for on-chip crystallization of lysozyme with the microdialysis method. The lysozyme crystals grown on-chip with the conditions provided in the second line were used for in situ X-ray diffraction data collection. 


\begin{tabular}{|c|c|c|c|}
\hline Protein & Lysozyme & Lysozyme & Lysozyme \\
\hline Number of crystals & 1 & 1 & 2 \\
\hline Number of diffraction frames & 40 & 30 & 70 \\
\hline Oscillation $\left({ }^{\circ}\right)$ per exposure & 1 & 1 & \\
\hline Exposure time (s) & 30 & 30 & \\
\hline Temperature (K) & 293 & 293 & 293 \\
\hline Space group & $\mathrm{P}_{4}{ }^{2}{ }_{12}$ & $\mathrm{P}_{4}{ }^{2}{ }_{12}$ & $\mathrm{P}_{4}{ }_{2}{ }_{12}$ \\
\hline Unit cell parameters & $\begin{array}{c}78.8678 .8637 .87 \\
90.090 .090 .0\end{array}$ & $\begin{array}{c}79.1779 .1737 .95 \\
90.090 .090 .0\end{array}$ & $\begin{array}{c}78.4778 .4737 .65 \\
90.090 .090 .0\end{array}$ \\
\hline Resolution range $(\AA)$ & $\begin{array}{l}27.31-1.95 \\
(2.02-1.95)\end{array}$ & $\begin{array}{l}27.39-1.96 \\
(2.03-1.96)\end{array}$ & $\begin{array}{l}27.17-1.85 \\
(1.91-1.85)\end{array}$ \\
\hline Mosaicity $\left({ }^{\circ}\right)$ & 0.319 & 0.121 & \\
\hline Total reflections (observed) & $25127(3552)$ & $19991(3001)$ & \\
\hline Unique reflections (observed) & $8641(1357)$ & $8295(1321)$ & $10404(975)$ \\
\hline Redudancy & $2.90(2.61)$ & $2.41(2.27)$ & \\
\hline Completeness (\%) & $95.0(94.8)$ & $91.9(93.3)$ & $98.23(93.15)$ \\
\hline 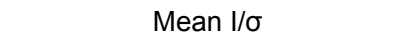 & $6.83(1.16)$ & $7.09(1.66)$ & 3.7 \\
\hline $\mathrm{CC}_{(1 / 2)}$ & $99.1(42.4)$ & $97.9(37.0)$ & 97.0 \\
\hline R-merge & & & 0.184 \\
\hline R-meas & 0.139 & 0.221 & 0.219 \\
\hline R-pim & & & 0.116 \\
\hline Reflections used in refinement & $8645(787)$ & $8451(857)$ & $10391(965)$ \\
\hline Reflections used for R-free & $864(78)$ & $846(85)$ & $1039(96)$ \\
\hline R-work & $0.1988(0.2968)$ & $0.1853(0.2872)$ & $0.1839(0.3102)$ \\
\hline R-free & $0.2430(0.3437)$ & $0.2297(0.3622)$ & $0.2207(0.3703)$ \\
\hline Number of non-hydrogen atoms & 1069 & 1071 & 1096 \\
\hline macromolecules & 1012 & 1012 & 1012 \\
\hline water & 55 & 57 & 82 \\
\hline
\end{tabular}




\begin{tabular}{|c|c|c|c|}
\hline ligand & 2 & 2 & 2 \\
\hline Protein residues & 131 & 131 & 131 \\
\hline Rms (bonds, $\AA$ ) & 0.008 & 0.009 & 0.005 \\
\hline Rms (angles, ${ }^{\circ}$ ) & 1.17 & 1.26 & 1.05 \\
\hline Ramachandran favored (\%) & 98.43 & 97.64 & 99.21 \\
\hline Ramachandran allowed (\%) & 1.57 & 2.36 & 0.79 \\
\hline Ramachandran outliers (\%) & 0.00 & 0.00 & 0.00 \\
\hline Avegare B-factor & 34.26 & 28.54 & 24.34 \\
\hline protein & 33.94 & 28.14 & 23.62 \\
\hline water & 40.23 & 35.57 & 33.16 \\
\hline ligands & 33.23 & 29.63 & 24.77 \\
\hline
\end{tabular}

Table 2: Data collection parameters, crystallographic and refinement statistics of lysozyme crystals grown onchip via the microdialysis method. Values provided in parentheses correspond to the highest resolution shell. The fourth column corresponds to values obtained after merging the data sets of the second and third column.

\section{Discussion}

A microfluidic device has been developed for on-chip protein crystallization with the microdialysis method and in situ X-ray diffraction experiments at room temperature. NOA 81 chips integrating RC dialysis membranes of any MWCO in order to use microdialysis for on-chip protein crystallization can be fabricated. Fabrication materials with a relatively high Xray transparency were used, rendering the chips compatible for in situ protein crystallography. The fabrication materials that compose the compartment for protein crystallization of the device (PMMA, Kapton, RC dialysis membrane) were evaluated to generate low background noise. Specifically, the background noise generated by the dialysis chip is mainly observed at low resolution $(>6 \AA)$ and does not affect the treatment of high-resolution diffraction data of the large lysozyme crystals required for protein structure determination. The automation of the data collection is amplified using a 3D printed support that can be mounted directly in macromolecular crystallography beamlines and carry up to three microchips simultaneously. This way, manual harvesting and manipulation of the fragile protein crystals is avoided. Moreover, the data collection takes place at room temperature, avoiding the need for cryoprotection which can be related to conformational changes from the native protein structure 2,3 .

The use of microdialysis as a method to grow crystals on-chip allows to accurately monitor and control the crystallization process. As discussed in the introduction, most of the conventional protein crystallization methods have been implemented using microfluidic devices ${ }^{11,14}$. However, the advantages of dialysis for protein crystallization had not yet been fully exploited at the microscale. On-chip microdialysis 
provides the possibility to study phase diagrams and perform screening and optimization of crystallization conditions with the same protein sample ${ }^{19}$. For the prototypes presented in this work, the protein consumption per chip is limited down to 0.1 or $0.3 \mu \mathrm{L}$. Based on the experimental work so far, the most critical steps of the protocol do not derive from the chips' fabrication procedure but from the crystallization process. The fabrication protocol includes many steps but it is straightforward and enables the fabrication of numerous devices (20 to 30 chips) in a single day in the clean room, with relatively inexpensive materials. However, the on-chip crystallization of proteins can be a delicate procedure due to the intrinsic stochastic nature of nucleation and crystal growth, especially in the microscale. A case study has been described, where well-established conditions were used for the crystallization of lysozyme that yielded robust, welldefined crystals suitable for in situ X-ray diffraction data collection. Nevertheless, difficulties may arise by the use of more challenging protein targets, such as membrane proteins, where the crystallization medium is much more complicated, phase diagrams are not known and well-working crystallization conditions are not yet well established. The dialysis chip offers the possibility to surpass these difficulties and study phase diagrams on-chip, without disposing the valuable and frequently costly protein sample, by merely exchanging the crystallization solution within the microfluidic channel.

The versatility of the microfluidic devices stems from exploiting microdialysis for on-chip protein crystallization in order to reversibly control crystallization conditions and map concentration and temperature varied phase diagrams using low protein volume. Moreover, the device is compatible with in situ X-ray diffraction experiments and the prototyping of the devices is inexpensive and rapid. Numerous, isomorphous crystals of soluble and membrane proteins (in preparation) can be grown on-chip and it is expected that all these features can be utilized for serial X-ray crystallography studies of challenging protein targets at synchrotron and XFEL facilities. Finally, performing on-chip and in situ time-resolved studies is a future possibility that could be of significant interest to the crystallographic community. Therefore, by growing crystals on the dialysis chip and introducing the reagents into the microfluidic channel, either manually (using a syringe) or automatically (with a pressure-control fluid system or a syringe-pump), future efforts will focus on proving that the microfluidic chips can be successfully used to trigger timeresolved experiments on synchrotron beamlines.

\section{Disclosures}

The authors have nothing to disclose.

\section{Acknowledgments}

MBS acknowledges the support of the MI / CNRS under the contract Instrumentation at the limits 2014-2015. NJ acknowledges CEA's International Doctoral Research Program (Irtelis) for the PhD Fellowship. MBS and SJ acknowledge funding from the European Union's Horizon 2020 Research and Innovation Programme under Marie Skłodowska-Curie grant agreement number 722687. MBS, SJ, and NJ thank LIPhy (UGA) for the clean room establishment for microfabrication experiments. IBS acknowledges integration into the Interdisciplinary Research Institute of Grenoble (IRIG, CEA).

\section{References}

1. Garman, E. F. Radiation damage in macromolecular crystallography: what is it and why should we care? Acta 
Crystallographica, Section D: Biological Crystallography. 66, 339-351 (2010).

2. Henderson, R. The potential and limitations of neutrons, electrons and X-rays for atomic resolution microscopy of unstained biological molecules. Quarterly Reviews of Biophysics. 28 (2), 171-193 (1995).

3. Fraser, J. S. et al. Accessing protein conformational ensembles using room-temperature $\mathrm{X}$ ray crystallography. Proceedings of the National Academy of Sciences of the United States of America. 108 (39), 16247-16252 (2011).

4. Gotthard, G. et al. Specific radiation damage is a lesser concern at room temperature. IUCrJ. 6 (4), 665-680 (2019).

5. Martin-Garcia, J. M., Conrad, C. E., Coe, J., RoyChowdhury, S., Fromme, P. Serial femtosecond crystallography: A revolution in structural biology. Archives of Biochemistry and Biophysics. 602, 32-47 (2016).

6. Gicquel, Y. et al. Microfluidic chips for in situ crystal X-ray diffraction and in situ dynamic light scattering for serial crystallography. Journal of Visualized Experiments: JoVE. 134, e57133 (2018).

7. Chapman, H. N. et al. Femtosecond X-ray protein nanocrystallography. Nature. 470, 73-78 (2011).

8. Hunter, M. S. et al. Fixed-target protein serial microcrystallography with an x-ray free electron laser. Science Reports. 4, 6026 (2014).

9. Pawate, A. S. et al. Towards time-resolved serial crystallography in a microfluidic device. Acta Crystallographica, Section F: Structural Biology Communications. 71, 823-830 (2015).
10. Owen, R. L. et al. Low-dose fixed-target serial synchrotron crystallography. Acta Crystallographica, Section D: Structural Biology. 73, 373-378 (2017).

11. Leng, J., Salmon, J.-B. Microfluidic crystallization. Lab on a Chip. 9, 24-34 (2009).

12. Morel, M., Galas, J.-C., Dahan, M., Studer, V. Concentration landscape generators for shear free dynamic chemical stimulation. Lab on a Chip. 12, 1340-1346 (2012).

13. Miralles, V., Huerre, A., Malloggi, F., Jullien, M.-C. A review of heating and temperature control in microfluidic systems: techniques and applications. Diagnostics. 3, 33-67 (2013).

14. Sui, S., Perry, S. L. Microfluidics: from crystallization to serial time-resolved crystallography. Structural Dynamics. 4, 032202 (2017).

15. Hansen, C. L., Sommer, M. O. A., Quake, S. R. Systematic investigation of protein phase behavior with a microfluidic formulator. Proceedings of the National Academy of Sciences of the United States of America. 101. (40), 14431-14436 (2004).

16. Laval., P., Lisai, N., Salmon, J.-B., Joanicot, M. A microfluidic device based on droplet storage for screening solubility diagrams. Lab on a Chip. 7, 829-834 (2007).

17. Shim, J.-Uk et al. Control and measurement of the phase behavior of aqueous solutions using microfluidics. Journal of the American Chemical Society. 129, 8825-8835 (2007).

18. Selimovic, S., Gobeaux, F., Fraden, S. Mapping and manipulating temperature-concentration phase 
diagrams using microfluidics. Lab on a Chip. 10, 1696-1699 (2010).

19. Junius, N. et al. A microfluidic device for both on-chip dialysis protein crystallization and in situ X-ray diffraction.

Lab on a Chip. 20, 296-310 (2020).

20. Greaves, E. D., Manz, A. Towards on-chip X-ray analysis. Lab on a Chip. 5, 382-391 (2005).

21. Dhouib, K. et al. Microfluidic chips for the crystallization of biomacromolecules by counter-diffusion and on-chip crystal X-ray analysis. Lab on a Chip. 9, 1412-1421 (2009).

22. Guha, S., Perry, S. L., Pawate, A. S., Kenis, P. J. A. Fabrication of X-ray compatible microfluidic platforms for protein crystallization. Sensors and Actuators $B$. Chemical. 174, 1-9 (2012).

23. Sui, S. et al. Graphene-based microfluidics for serial crystallography. Lab on a Chip. 16, 3082-3096 (2016).

24. Russo Krauss, I., Merlino, A., Vergara, A., Sica, F. An overview of biological macromolecule crystallization. International Journal of Molecular Science. 14., 11643-11691 (2013).

25. McPherson, A., Gavira, J. A. Introduction to protein crystallization. Acta Crystallographica, Section F: Structural Biology and Crystallization Communications. 70, 2-20 (2014).

26. Zheng, B., Tice, J. D., Roach, L. S., Ismagilov, R. F. A droplet-based, composite PDMS/Glass capillary microfluidic system for evaluating protein crystallization conditions by microbatch and vapor-diffusion methods with on-chip X-ray diffraction. Angewandte Chemie (International Ed.ition in English) 43, 2508-2511 (2004).
27. Talreja, S., Kim, D. Y., Mirarefi, A. Y., Zukoski, C. F., Kenis, P. J. A. Screening and optimization of protein crystallization conditions through gradual evaporation using anovel crystallization platform. Journal of Applied Crystallography. 38, 988-995 (2005).

28. Hansen, C. L., Classen, S., Berger, J. M., Quake, S. R. A microfluidic device for kinetic optimization of protein crystallization and in situ structure determination. Journal of American Chemical Society. 128, 3142-3143 (2006).

29. Schieferstein, J. M. et al. X-ray Transparent microfluidic platforms for membrane protein crystallization with microseeds. Lab on a Chip. 18, 944-954 (2018).

30. Ghazal, A. et al. Recent advances in X-ray compatible microfluidics for applications in soft materials and life sciences. Lab on a Chip. 16, 4263-4295 (2016).

31. Li, L., Ismagilov, R. F. Protein crystallization using microfluidic technologies based on valves, droplets, and SlipChip. Annual Review of Biophysics. 39, 139-158 (2010).

32. Du, W., Li, L., Nichols, K. P., Ismagilov, R. F. SlipChip. Lab on a Chip. 9, 2286-2292 (2009).

33. Zhang, S. et al. Microfluidic platform for optimization of crystallization conditions. Journal of Crystal Growth. 472 , 18-28 (2017).

34. Abdallah, B. G. et al. Protein crystallization in an actuated microfluidic nanowell device. Crystal Growth \& Design. 16, 2074-2082 (2016).

35. Monteiro, D. C. F. et al. A microfluidic flow-focusing device for low sample consumption serial synchrotron crystallography experiments in liquid flow. Journal of Synchrotron Radiation. 26, 406-412 (2019). 
36. de Wijn, R. et al. A simple and versatile microfluidic device for efficient biomacromolecule crystallization and structural analysis by serial crystallography. IUCrJ. 6 , 454-464 (2019).

37. Shim, J.-Uk, Cristobal, G., Link, D. R., Thorsen, T., Fraden, S. Using microfluidics to decouple nucleation and growth of protein crystals. Crystal Growth \& Design. 7., 2192-2194 (2007).

38. de Jong, J., Lammertink, R. G. H., Wessling, M. Membranes and microfluidics: a review. Lab on a Chip. 6, 1125-1139 (2006).

39. Paustian, J. S., Nery Azevedo, R., Lundin, S.-T. B., Gilkey, M. J., Squires, T. M. Microfluidic microdialysis: spatiotemporal control over solution microenvironments using integrated hydrogel membrane microwindows. Physical Review X. 3, 041010 (2013).

40. Kornreich, M., Heymann, M., Fraden, S., Beck, R. Cross polarization compatible dialysis chip. Lab on a Chip. 14, 3700-3704 (2014).

41. Song, S., Singh, A. K., Shepodd, T. J., Kirby, B. J. Microchip dialysis of proteins using in situ photopatterned nanoporous polymer membranes. Analytical Chemistry. 76, 2367-2373 (2004).

42. Skou, M., Skou, S., Jensen, T. G., Vestergaard, B., Gillilan, R. E. In situ microfluidic dialysis for biological small-angle X-ray scattering. Journal of Applied Crystallography. 47, 1355-1366 (2014).

43. Zou, L. et al. A multistage dialysis microdevice for extraction of cryoprotectants. Biomedical Microdevices. 19, 30 (2017).

44. Satya Eswari, J., Naik, S. A critical analysis on various technologies and functionalized materials for manufacturing dialysis membranes. Materials Science for Energy Technologies. 3, 116-126 (2020).

45. Spano, M., Salmon, J.-B., Junius, N. FR3044685A1. UJF, (2015).

46. Bartolo, D., Degre, G., Nghe, P., Studer, V. Microfluidic stickers. Lab on a Chip. 8, 274-279 (2008).

47. Junius, N. et al. A crystallization apparatus for temperature controlled flow-cell dialysis with real-time visualization. Journal of Applied Crystallography. 49, 806-813 (2016).

48. Kabsch, W. XDS. Acta Crystallographica, Section D: Biological Crystallography. 66, 125-132 (2010).

49. Winn, M.D. et al. Overview of the CCP4 suite and current developments. Acta Crystallographica, Section D: Biological Crystallography. 67, 235-242 (2011).

50. Emsley, P., Lohkamp, B., Scott, W. G., Cowtan, K. Features and developments of COOT. Acta Crystallographica, Section D: Biological Crystallography. 66, 486-501 (2010).

51. Apostolopoulou, V., Junius, N., Sear, R. P., BudayovaSpano, M. Mixing salts and polyethylene glycol into protein solutions: The effects of diffusion across semipermeable membranes and of convection. Crystal Growth \& Design. 20, 3927-3936 (2020).

52. Liebschner, D. et al. Macromolecular structure determination using X-rays, neutrons and electrons: recent developments in Phenix. Acta Crystallographica, Section D: Structural Biology. 75, 861-877 (2019).

53. Xia, Y., Whitesides, G.M. Soft Lithography. Annual Review of Materials Science. 28, 153-84 (1998).

54. Nogly, P. et al. Lipidic cubic phase injector is a viable crystal delivery system for time-resolved serial 
crystallography. Nature Communications. 7, 12314 (2016).

55. Baxter, E. L. et al. High-density grids for efficient data collection from multiple crystals. Acta Crystallographica, Section D: Structural Biology. 72., 2-11 (2016).

56. Feiler, C. G., Wallacher, D., Weiss, M. S. An all-in-one sample holder for macromolecular X-ray crystallography with minimal background scattering. Journal of Visualized Experiments. 149., e59722 (2019). 